\title{
Identification of Shocks in the Spectra from Black Holes
}

\author{
Samir Mandal (space_phys@vsnl.com) \\ Centre for Space Physics, Chalantika 43, Garia Station Rd., Kolkata 700084 \\ Sandip Kumar Chakrabarti (chakraba@bose.res.in) \\ S.N. Bose National Centre for Basic Sciences, JD Block, Salt Lake, Kolkata \\ 700098 \\ and \\ Centre for Space Physics, Chalantika 43, Garia Station Rd., Kolkata 700084
}

August 26th, 2004

\begin{abstract}
We study the spectral properties of a low angular momentum flow as a function of the shock strength, compression ratio, accretion rate and flow geometry. In the absence of a satisfactory description of magnetic fields inside the advective disk, we consider the presence of only stochastic fields and use the ratio of the field energy to the gravitational energy density as a parameter. We not only include 'conventional' synchrotron emission and Comptonization by MaxwellBolzmann electrons in the gas, but we also compute these effects due to power-law electrons. For strong shocks, a bump is produced due to the post-shock flow. A power-law spectral components due to the thermal and non-thermal electrons appear after this bump.
\end{abstract}

Keywords: Black hole physics — shocks — hydrodynamics — spectral properties: $\gamma$ rays, X-rays

To appear in Astrphysics and Space Sciecne (Proceeding of the Hong Kong Conference; edited by K.S. Cheng and Romero)

\section{Introduction}

In Chakrabarti \& Titarchuk (1995, hereafter CT95), a two component advective flow (TCAF) solution was introduced (see, Chakrabarti, this volume). The sub-Keplerian flow has a steady shock but depending on the flow parameters it could be oscillating or propagating. The postshock region is called CENtrifugal pressure supported BOundary Layer or CENBOL. Given that the accretion flow largely passes through this shock, it is likely that the hot electrons may be accelerated by the shock just as high energy cosmic rays are produced by the transient supernovae shocks (Bell 1978ab, Longair 1981). We assume this process in computing the spectra and show that the presence of shocks make their mark distinctly. Our solution also produces gamma-rays naturally since shocks are generally produced due to the centrifugal barrier (e.g., Chakrabarti, 1996). It is possible that occasionally an additional source of high energy photons, perhaps in the form of shocks in outflows and

(C) 2018 Kluwer Academic Publishers. Printed in the Netherlands.

shockidenti.tex; 15/06/2018; $17: 33 ;$ p.1 
jets, is necessary to explain the complete spectra. Details of our model are presented in Mandal \& Chakrabarti (2004).

\section{Relevant heating and cooling processes and the solution procedure}

Protons loss energy through Coulomb interaction and inverse bremss -trahlung. Electrons are heated through this Coulomb coupling. Electrons are cooled by bremsstrahlung, cyclo-synchrotron and Comptonization of the soft photons due to cyclo-synchrotron radiation. The Comptonization is computed by using this cooling term augmented by the enhancement factor. In the present situation, the flow passes through the shocks and thus the electrons will have a power-law distribution Thus, both the thermal and the non-thermal electrons are present.

The geometry of the flow is chosen to be conical for simplicity and the flow surface makes an angle $\Theta$ with the z-axis. We assumed the form of infall velocity $v(x)=v_{0} x^{-1 / 2}$. At the shock, $x=x_{\mathrm{s}}$, the velocity is reduced by a factor $R$, the compression ratio, i.e., $v_{+}\left(x_{\mathrm{s}}\right)=v_{-}\left(x_{\mathrm{s}}\right) / R$. The number density also goes up by a factor $R$.

As for the initial conditions, we fix the outer boundary at a large distance (say, $10^{6} r_{\mathrm{g}}$ ) and supply matter (both electrons and protons) with the same temperature (say, $T_{\mathrm{p}}=T_{\mathrm{e}}=10^{6} \mathrm{~K}$ ). By integrating energy equation, we obtained the temperature. The radiation emitted by the flow through bremsstrahlung and synchrotron radiation are inverse Comptonized by the hot electrons in the flow.

A shock of compression ratio $R$ causes the formation of power-law electrons of slope (Bell, 1978ab), $p=(R+2) /(R-1)$. This powerlaw electrons produce a power-law synchrotron emission with an index (Longair, 1981) $q=(1-p) / 2$. The power-law electrons have energy

minimum at $\mathcal{E}_{\text {min }}$ and energy maximum at $\mathcal{E}_{\text {max }}$. $\mathcal{E}_{\text {max }}$ is obtained selfconsistently by conserving the number of power-law electrons and by computing the number of scatterings that the electrons undergo inside the disk before they escape and $\mathcal{E}_{\text {min }}$ is obtained from the temperature of the injected electrons. This temperature is obtained self-consistently through our integration procedure.

In presence of magnetic field (assumed to be the ratio of the magnetic energy density to gravitational energy density), the pre-shock flow and the CENBOL would emit synchrotron radiation. We assume that the number of black body photons generated by the electrons obeying power-law distribution (assumed to be of constant fraction $\zeta$ of thermal electrons) below self-absorption frequency is the same fraction $\zeta$ of the total black body photons. We followed the procedures 

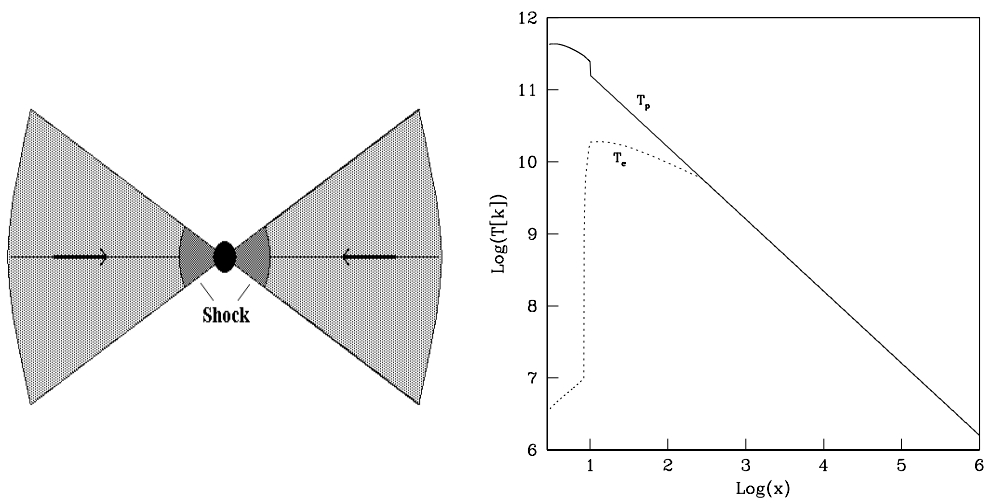

Figure 1. (left) Wedge shaped flow model assumed in this paper which included a shock at the inner region. (right) Variation of proton and electron temperatures (marked as $T_{\mathrm{p}}$ and $T_{\mathrm{e}}$ ) as functions of dimensionless radial distance $x$ for $\dot{m}=0.00059$. Other parameters are $M=10 M_{\odot}, R=3.9, \Theta=77^{\circ}, \zeta=0.7$ and $x_{\mathrm{s}}=10$. Closer to the black hole, the electrons cool more rapidly than the protons.

presented in CT95 and Titarchuk \& Lyubarskij (1995) while computing the Comptonized spectrum due to scattering from thermal electrons and power-law electrons. However, unlike CT95 where a Keplerian disk was supplying soft photons, here the source is everywhere, i.e., distributed throughout. This has been taken into account. At the end, we add contributions from all the components to get the net photon emission from the flow.

In the next Section, we present the spectral characteristics by varying parameters such as $\Theta, x_{\mathrm{s}}, R, \zeta$ and $\dot{m}$. We use a black hole of mass $10 M_{\odot}$.

\section{Results and interpretations}

In Fig. 1 we show our simplified flow geometry to capture the essential physics and show how the temperature of the electrons and protons differ as the flow moves closer to the black hole. The deviation is more prominent in the post-shock region. Here, the radial distance $x$ is measured in units of the Schwarzschild radius $r_{\mathrm{g}}$.

In Fig. 2, we show a typical spectrum obtained from our solution. The curve marked 1 is due to pre-shock flow and the curve marked 2 is due to the post-shock flow. The synchrotron self-absorption frequency at $\nu_{\mathrm{a}}$ is marked. The bump due to post-shock flow is at frequency $\nu_{\text {bump }} \sim \nu_{\text {inj }}\left[1+\frac{4}{3} \frac{R-1}{R} \frac{1}{x_{r m s}^{1 / 2}}\right]^{x_{\mathrm{s}}^{1 / 2}}$, where, $\nu_{\text {inj }}$ is the frequency of the 


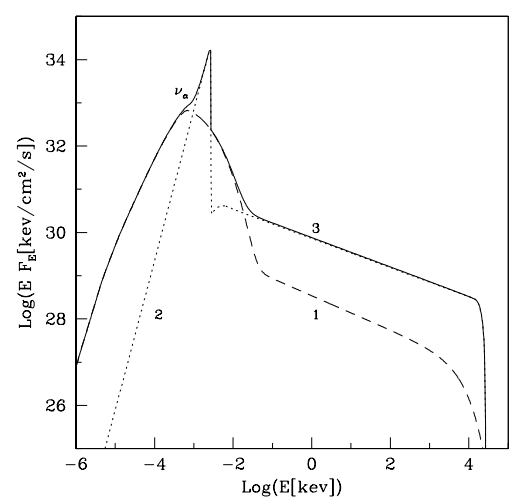

Figure 2. A typical complete spectrum from a sub-Keplerian accretion flow with all the contributions shown for the same parameters as in Fig. 1b. The curve marked ' 1 ' is due to Comptonization of the synchrotron emission from the pre-shock region. The curve marked ' 2 ' is due to the Comptonized spectra from the post-shock (CENBOL) region. The curve marked ' 3 ' indicates the net radiation from the flow. $\nu_{\text {a }}$ represents synchrotron self-absorption frequency for the CENBOL.

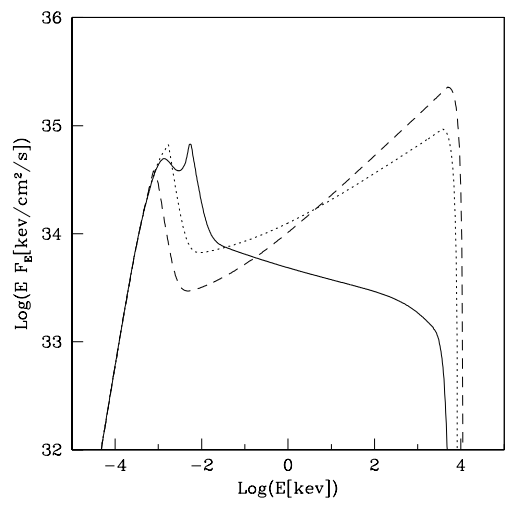

Figure 3. Variation of the total emitted spectrum when the shock location $x_{\mathrm{s}}$ is varied. The solid, dotted and dashed curves are drawn for $x_{\mathrm{s}}=10,40,80$ respectively. The spectrum becomes softer when $x_{\mathrm{s}}$ is reduced. Here, $\dot{m}=0.1$ has been chosen.

dominant photons from the pre-shock flow, $R$ is the compression ratio of the shock located at $x_{\mathrm{s}}$. The power law is produced by the power-law electrons from the shock acceleration.

In Fig. 3, we compare three cases with shock locations at $x_{\mathrm{s}}=10,40$ and 80 respectively. The shock locations can vary with specific angular momentum in the flow which in turn is governed by the viscosity parameter (Chakrabarti, 1990; 1996).

With the rise in the accretion rate of the sub-Keplerian disk, the spectrum is likely to become harder. This can be seen more clearly in Fig. 4, where we vary the accretion rate. A higher accretion rate is 


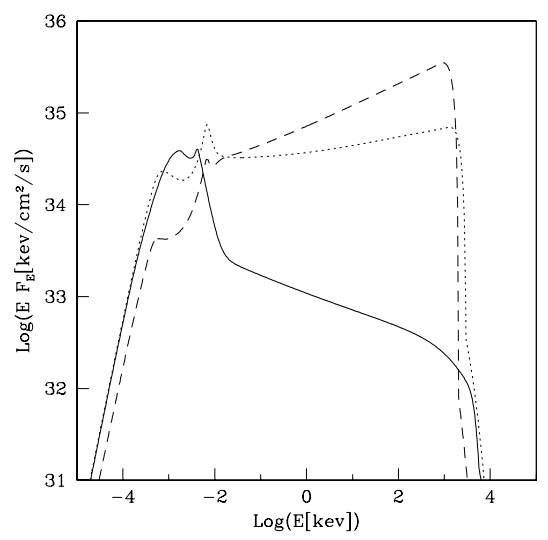

Figure 4. Variation of the spectra with the dimensionless accretion rate. Solid, dotted and dashed curved are for $\dot{m}=0.05,0.5$ and 1.5 respectively. The spectrum becomes harder when the accretion rate is increased.

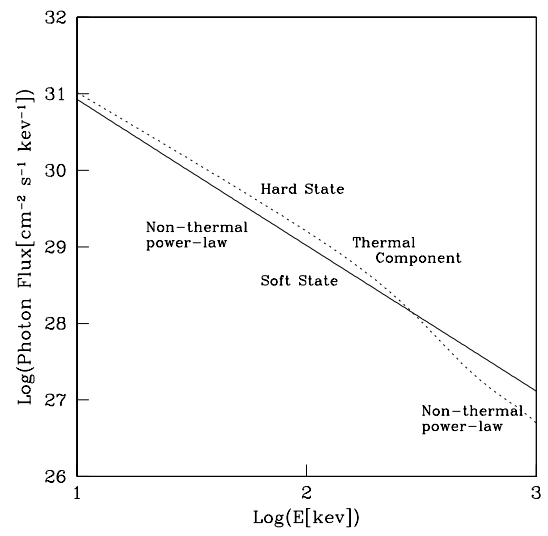

Figure 5. Example of a typical soft state and a hard state spectra of a galactic black hole candidate os mass $10 M_{\odot}$. The parameters for the hard state are: $\dot{m}=2.4$, $R=4, x_{\mathrm{s}}=250, \Theta=80, \zeta=0.24$. The parameters for the soft state are: $\dot{m}=0.02$, $R=4, x_{\mathrm{s}}=100, \Theta=80, \zeta=0.3$.

expected to increase the density of the flow and it becomes difficult to cool the matter by the soft photons of the synchrotron radiation. The Comptonized spectrum becomes harder. At lower accretion rate, it is easy to cool the flow and the spectrum becomes softer. The curves are drawn for: $\dot{m}=0.05$ (solid), 0.5 (dotted) and 1.5 (dashed). 


\section{A typical soft/hard spectra in gamma-ray region}

Typical broad band soft state and hard state spectra are presented in Fig. 4 of Chakrabarti (this volume). In Fig. 5 of Chakrabarti (this volume) behaviour at high energy has been shown for Cyg X-1, GROJ171924 and GROJ0422+32 (taken from Ling \& Wheaton, 2004). In Fig. 5 we present the photon flux in soft and hard states as obtained by our solution with shocks. No jet contribution was added. Not surprisingly, they look similar. However, a second kind of high $\gamma$ intensity state was reported (see, Case et al. 2004) in which non-thermal power-law has been observed from a few tens of keV to few MeVs (see, Fig. 4 of Chakrabarti, this volume). It has been difficult to obtain such a behaviour without invoking jets or other mechanisms.

\section{Acknowledgements}

SM acknowledges a RESPOND project from Indian Space Research Organization which supports his research.

\section{References}

Bell, A.R., The acceleration of cosmic rays in shock fronts. I, MNRAS, 182, 147, 1978a.

Bell, A.R., The acceleration of cosmic rays in shock fronts. II MNRAS, 182, 443, 1978b.

Case, G. L. et al., Observations of gamma-ray outbursts from galactic microquasars, Chinese J. Astron. Astrophys. (in press)

Chakrabarti, S. K., Theory of transonic astrophysical flows, World Scientific Publishing Co., Singapore, 1990.

Chakrabarti, S. K., Grand unification of solutions of accretions and winds around black holes and neutron stars, Astrophys. J., 464, 664, 1996.

Chakrabarti, S. K., Acharyya, K. \& Molteni, D., The effect of cooling on time dependent behaviour of accretion flows around black holes, Astronom. E Astrophys., 421, 1, 2004.

Chakrabarti, S. K., Manickam, S. G., Correlation among quasi-Periodic oscillation frequencies and quiescent-state duration in black hole candidate GRS 1915+105, Astrophys. J. 531, L41, 2000.

Chakrabarti, S. K. \& Titarchuk, L.G., Spectral properties of galactic and extragalactic black hole candidates, Astrophys. J., 455, 623, 1995.

Ling, J. C. \& Wheaton, W. A., Gamma-ray spectral characteristics of thermal and non-thermal emission from three black holes, Chinese J. Astron. Astrophys. (in press)

Longair, M. S., High energy astrophysics, Cambridge Univ. Press, UK, 1981.

Mandal, S. \& Chakrabarti, S. K. Signature of accretion shocks in emitted radiation from a two temperature advective flows around black holes, Astron. $\&$ Astrophys., (submitted) 
Titarchuk, L.G., Lyubarskij, Y., Power-law spectra as a result of comptonization of the soft radiation in a plasma cloud, Astrophys. J., 450, 876, 1995. 
shockidenti.tex; 15/06/2018; $17: 33 ;$ p.8 